Minari, T., et al. Experiments on Condensed Matter Nuclear Events in Kobe University. in Eleventh International Conference on Condensed Matter Nuclear Science. 2004. Marseille, France.

\title{
Experiments on Condensed Matter Nuclear Events in Kobe University
}

T. Minari, R. Nishio, A. Taniike, Y. Furuyama and A. Kitamura*

Division of Environmental Energy Science, Graduate School of Science and Technology, Kobe University, Japan

5-1-1 Fukaeminami-machi, Higashinada-ku, Kobe 658-0022, Japan

*kitamura@maritime.kobe-u.ac.jp

\begin{abstract}
We review three kinds of experimental work underway in our laboratory to investigate nuclear events in solid or liquid materials. The largest effort has been given to experiments to confirm the ${ }^{7} \mathrm{Li}(\mathrm{d}, \mathrm{n} 2 \alpha)$ reaction rate enhancement reaching $10^{15}$ in liquid lithium which was reported by H. Ikegami et al. [4] Li liquid droplets are formed as targets, and to keep them as pure as possible, we built a liquid Li loop. Thus far, in all cases of irradiation at the temperature from 520 to $570 \mathrm{~K}$ with $10-24 \mathrm{keV}$ deuterons, we have not been able to reproduce the Ikegami enhancement for the ${ }^{7} \operatorname{Li}(\mathrm{d}, \mathrm{n} 2 \alpha)$ reaction.
\end{abstract}

\section{Introduction}

Experimental studies on condensed matter nuclear reaction using ion beams at Kobe University are reviewed. First, we are continuing experiments on $\mathrm{keV}$-deuterium ion irradiation of deuterated $\mathrm{Au} / \mathrm{Pd}$ samples, searching for possible anomalous nuclear reactions in solids. [1,2] The Au-coated surface of the Pd sample is irradiated with the $\mathrm{keV}$-deuterium ions and with $\mathrm{MeV}$ ion beams for simultaneous characterization of the surface, while the rear surface is exposed to $D_{2}$ gas at atmospheric pressure to load the sample.

In addition, a modified version of the sample system without the implanter has been installed at another beam line of the accelerator exclusively in order to reproduce the so-called Iwamura effect. [3] Iwamura et al. observed transformation of ${ }^{133} \mathrm{Cs}$ into ${ }^{141} \mathrm{Pr}$ during forced permeation of deuterium through a multi-layered film of $\mathrm{Pd}$ and $\mathrm{CaO}$. In the present system of ours the nuclear transformation is monitored by in situ PIXE analysis during forced permeation of deuterium.

The third system has been installed to reproduce the experiments made by H. Ikegami et al.[4], in which they observed an enormous enhancement of ${ }^{7} \operatorname{Li}(\mathrm{d}, \mathrm{n} 2 \alpha)$ reaction rate in liquid lithium. To maintain high purity, we have set up a keV-deuteron irradiation system with a liquid Li loop, which enables us to continuously bombard pure $\mathrm{Li}$ in a closed container.

\section{2. $D(d, p) t$ Reaction Rate Enhancement}

The $\mathrm{D}(\mathrm{d}, \mathrm{p}) \mathrm{t}$ reaction rate under irradiation of deuterium ion beam has been measured in the keV energy range in various samples to determine whether the nuclear reaction rate can be enhanced in a condensed matter. Kasagi et al. reported that in $\mathrm{PdO}$, the enhancement factor relative to the reaction rate for the deuteron pair in vacuum increases up to fifty at $E_{\mathrm{d}}=2.5 \mathrm{keV}$, and that the screening energy deduced from the excitation function amounts to $600 \mathrm{eV}$. [5] 
We are continuing the experimental study on the $\mathrm{D}(\mathrm{d}, \mathrm{p}) \mathrm{t}$ reaction rate enhancement under deuterium ion irradiation of Au-deposited $\mathrm{Pd}(\mathrm{Au} / \mathrm{Pd})$ with in situ and simultaneous measurement of deuterium density by accelerator analyses. In this section, we report recent results. The details of the experimental procedure are described in previous papers [1,2].

In the latest experiments, the time of exposure of the 1-mm-thick $\mathrm{Au} / \mathrm{Pd}$ to deuterium gas was extended to $60 \mathrm{hrs}$ to make the deuterium density as high as possible. The results are summarized in Fig. 1 as a function of the center-of-mass energy $E_{\mathrm{c}}$ of the reacting pair. In this figure, the theoretical curves calculated with screening energy $U_{\mathrm{s}}[6]$ taken into account are also shown for comparison.

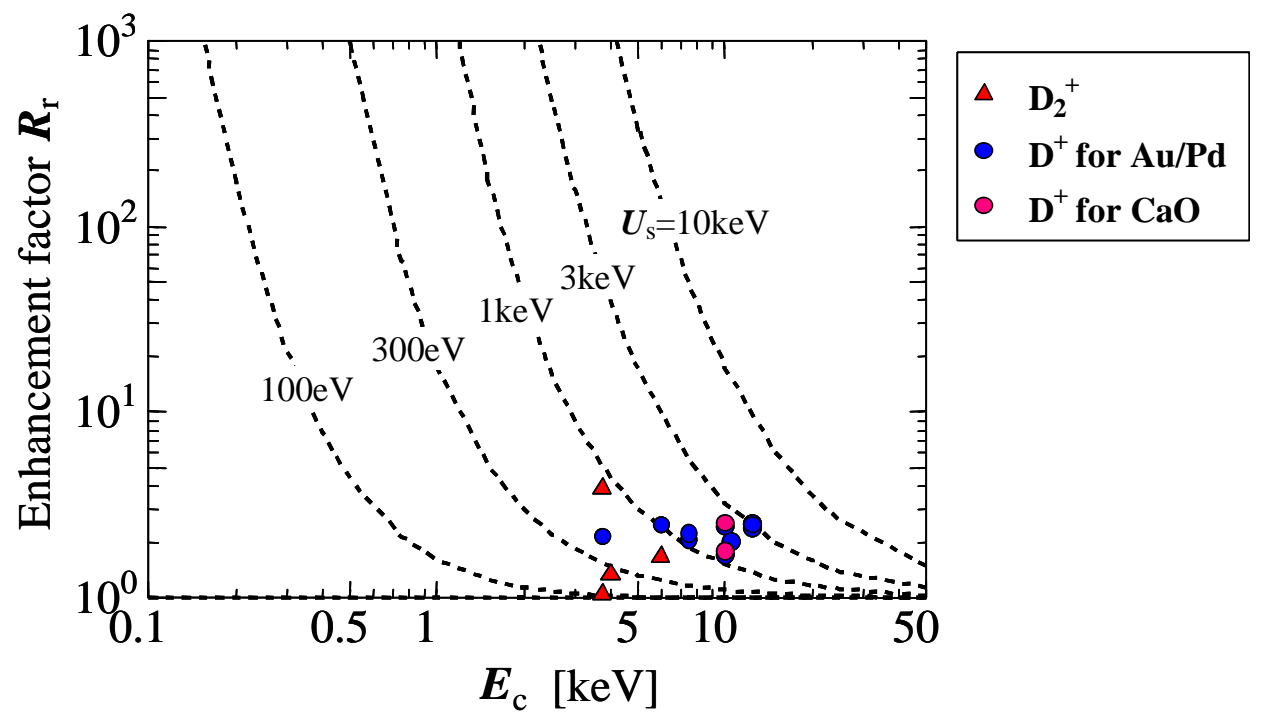

Fig. 1. Enhancement factor for $D(d, p) t$ reaction rate.

In the experiments by J. Kasagi et al., the value of $U_{\mathrm{s}}$ was found to be $250 \mathrm{eV}$ for the Pd bulk[7]. With our $\mathrm{Au} / \mathrm{Pd}$ sample, we have $U_{\mathrm{s}}$ of the order of $\mathrm{keV}$ in some cases, but with rather poor reproducibility. It seems that the enhancement factor depends on an unknown parameter which we have not controlled yet. Further experiments with purified conditions are needed to clarify the enhancement mechanism.

\section{PIXE Analysis of Palladium Complex under}

\section{$\mathrm{D}_{2}$ gas Permeation}

In Ref. 3 it is claimed that deuterium permeation through a $\mathrm{Cs}$ doped $\mathrm{Pd} /(\mathrm{CaO}+\mathrm{Pd}) / \mathrm{Pd}$ sample induced a transmutation from ${ }^{133} \mathrm{Cs}$ to ${ }^{141} \mathrm{Pr}$. In the present work, the experimental apparatus for PIXE analysis made in situ and simultaneously with gas permeation through samples has been built up.

Figure 2 shows a schematic of the experimental system. A sample with a $\mathrm{Pd}(\mathrm{Cs}) / \mathrm{CaO} / \mathrm{Pd}$ multilayer

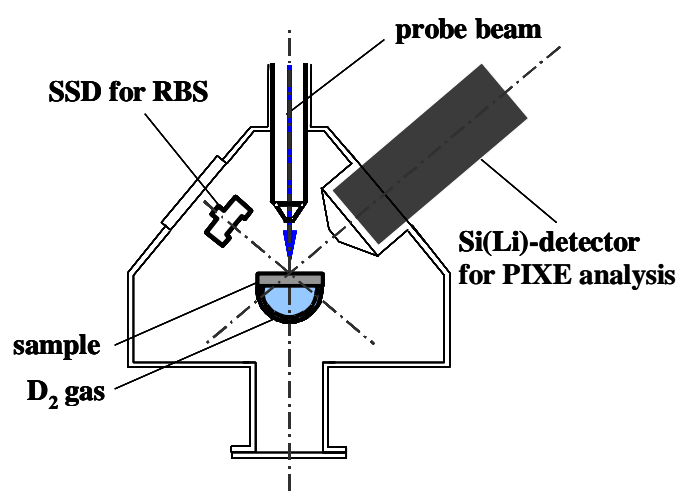

Fig. 2. Experimental system of PIXE analysis of metallic samples under gas permeation. 
on the surface is placed in a vacuum chamber, and its rear surface is exposed to $\mathrm{D}_{2}$ gas at a pressure of $0.1 \mathrm{MPa}$ typically. The multilayer surface is irradiated with probe beam ions to emit characteristic X-rays, which are analyzed with a lithium-drifted silicon $(\mathrm{Si}(\mathrm{Li})$ ) detector positioned at $135 \mathrm{deg}$. relative to the probe beam direction. Another solid-state detector (SSD) is provided at -135 deg. for RBS characterization of the sample.

The density $N$ of any target element can be evaluated from the X-ray yield $\Delta Y_{\mathrm{X}}$ during irradiation with $Q$ projectiles:

$$
\Delta Y_{\mathrm{X}}=Q N \Delta t \frac{\sigma_{i}(E)}{4 \pi} \omega_{\mathrm{K}} k_{\alpha} \Delta \Omega \exp \left(\frac{-\mu t}{\cos \theta}\right) T \varepsilon
$$

where $\sigma_{\mathrm{i}}(E), \omega_{\mathrm{K}}, k_{\alpha}$ and $\Delta \Omega$ denote the ionization cross section at a colliding energy of $E$, fluorescence yield for the K-shell, fractional transition probability of the $\mathrm{K}_{\alpha}$-line to all $\mathrm{K}$-lines and the solid angle of the detector, respectively. Then, $\mu, \theta, T$ and $\varepsilon$ are the X-ray absorption coefficient of the target itself, the detection angle, $\mathrm{X}$-ray transmittance of the absorbers in front of the detector and the detection efficiency of the detector, respectively. Here, homogeneous distribution of the element throughout the thickness $\Delta t$ is assumed.

A rough estimate shows that the minimum detectable areal densities of Pr and Cs are $4.3 \times 10^{14} \mathrm{~cm}^{-2}$ and $1.6 \times 10^{14} \mathrm{~cm}^{-2}$ for $100-\mathrm{p} \mu \mathrm{C} / 5-\mathrm{MeV} \alpha$ analysis. These limiting values of the areal densities have been confirmed by preliminary analyses of the $\mathrm{Au} / \mathrm{Pd}$ sample and a $\mathrm{CaO} / \mathrm{Pd}$ sample. Experiments using multilayered $\mathrm{Pd}(\mathrm{Cs}) / \mathrm{CaO} / \mathrm{Pd}$ samples are in progress.

\section{4. $\quad{ }^{7} \operatorname{Li}(d, n 2 \alpha)$ Reaction Rate Enhancement in Liquid Lithium}

An enormous enhancement of ${ }^{7} \mathrm{Li}(\mathrm{d}, \mathrm{n} 2 \alpha)$ reaction rate in liquid $\mathrm{Li}$ was reported by $\mathrm{H}$. Ikegami et al.[8]. They bombarded metallic Li targets in both liquid and solid phase with deuterium ion beams at the energy range of some tens of keV. In solid phase, no event was observed with the $\alpha$-particle and neutron detectors, which was consistent with the reaction rate estimation based on the published nuclear cross-section data. On the other hand, in the liquid phase, a large number of $\alpha$-particles were observed on the SSD. Consequently, the rate enhancement of ${ }^{7} \mathrm{Li}(\mathrm{d}, \mathrm{n} 2 \alpha)$ reaction was estimated to be a factor of $10^{10}-10^{15}$.

They explain the phenomenon as follows. Deuterons with keV energy ("buffer energy"), where the nuclear collision dominates over the electronic one in the stopping process, penetrate deep into the s-electron cloud of $\mathrm{Li}$ atoms to form "united atoms" [LiD] at the classical turning point. This means formation of the atomic fusion state which makes adiabatic transition to the nuclear fusion reaction with some probabilities determined by the well-known Gamow factor. Regarding the energetic deuterons as solutes, this process can be treated within the framework of thermodynamics of chemical reactions in dilute solutions. As a consequence the Arrhenius equation for spontaneous chemical reactions is applied. The Gibbs free energy change $\Delta G$ in the exponent of the enhancement factor is negative in the present case of endothermic reaction $\mathrm{d}+{ }^{7} \mathrm{Li} \rightarrow[\mathrm{LiD}]$. Thus an enhancement $\exp (-\Delta G / k T)$ by many orders of magnitude of nuclear fusion reactions in the metallic Li liquids could be realized.

In the present work, we investigate the reproducibility of the ${ }^{7} \operatorname{Li}(\mathrm{d}, \mathrm{n} 2 \alpha)$ reaction rate enhancement. The details of experimental procedure and experimental results are described below. 


\subsection{Experimental Procedure}

A schematic of the experimental system is shown in Fig. 3 and Fig. 4.

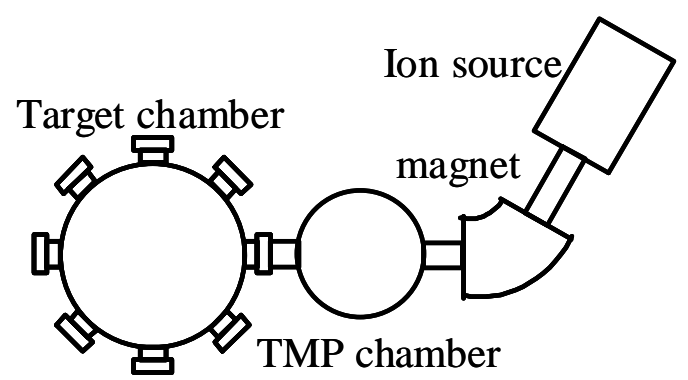

(a) Overall view

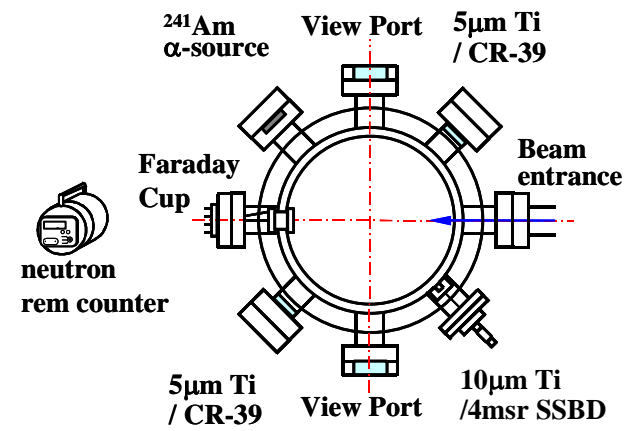

(b) Target chamber

Fig. 3. Plane view of the experimental svstem.

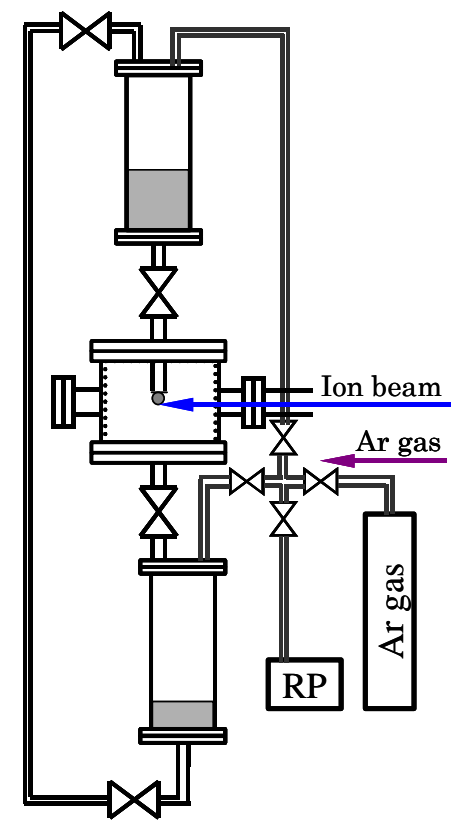

Fig. 4. Vertical view of the target chamber and the Liquid Li loop.

Beams of $20 \mathrm{keV}$ deuterons are extracted from a duoplasmatron ion source and mass-analyzed with a 60-deg. sector magnet. Deuterons are injected into the liquid $\mathrm{Li}$ target through an aperture of $10 \mathrm{~mm}$ in diameter. In the experiments made by $\mathrm{H}$. Ikegami et al., the detection of the $\alpha$-particles was frequently disturbed by hydroxide/nitride formation on the Li surface. To supply pure surface of liquid Li to the beam target, we have built a liquid Li loop in the present work.

A total of $400 \mathrm{~g}$ of $\mathrm{Li}$ is prepared in the upper reservoir. Liquid $\mathrm{Li}$ heated in the reservoir is dripped into a manifold through a 1/4-inch pipe to form spherical liquid droplets which are bombarded with deuterons at the center of the target chamber. The end of the pipe, from which the droplets fall into the beam, was located close to the beam to make the irradiation time as long as possible.

The temperature of $\mathrm{Li}$ is controllable to $570 \mathrm{~K}$ at the maximum so that the pipe does not get clogged. It takes about ten hours to finish dropping the total mass of $\mathrm{Li}$, which is divided into several thousand droplets. The irradiation time for one droplet is controlled by adjusting the conductance of the valve, and ranges from a few tens ms up to several minutes when a drop hangs on the outlet of the nozzle. For the total mass of $\mathrm{Li}$, the maximum irradiation time is several hours per a run, giving the total dose of $10^{16}$ protons. After one run $\mathrm{Li}$ is pumped from the bottom reservoir up to the upper reservoir by pressurizing the former with Ar gas.

During the run the beam current measured with a Faraday cup placed at the downstream end of the manifold is monitored with an oscilloscope. A current dip due to a droplet crossing the beam is a measure of the current flowing into the droplet. 
Alpha-particles are observed as the products of the ${ }^{7} \operatorname{Li}(\mathrm{d}, \mathrm{n} 2 \alpha)$ reaction using an SSD and some sheets of solid-state track detectors (CR-39). Neutrons are observed with a rem-counter. The solid angles are defined to $1.1-5.0 \times 10^{-3} \mathrm{sr}$ by the active area of the detector with a $5.6 \mathrm{~mm}$ diameter. During the first run, a $12.5-\mu \mathrm{m}-$ thick $\mathrm{Al}$ film was mounted on each detector to shield it from the $\mathrm{Li}$ vapor. But some dots introduced by scattered $\mathrm{Li}$ droplets were found in the film. To prevent melting, we changed the filter from $\mathrm{Al}$ to $10.0-\mu \mathrm{m}-\mathrm{thick} \mathrm{Ti}$. $\mathrm{A}^{241} \mathrm{Am}$ alpha source was located in the target chamber to allow calibration of the SSD simultaneously with the ion beam irradiation of the Li droplets.

\subsection{Data Analysis}

The enhancement of the ${ }^{7} \operatorname{Li}(\mathrm{d}, \mathrm{n} 2 \alpha)$ reaction rate could be confirmed by observation of enormous yields of $\alpha$-particles. H. Ikegami observed the $\alpha$-particles forming a single peak on the MCA with an energy shifting around 7.5 MeV, which is a half of the Q-value $(15.1 \mathrm{MeV})$ of the ${ }^{7} \mathrm{Li}(\mathrm{d}, \mathrm{n} 2 \alpha)$ reaction. All the counts in the ROI, $Y_{\alpha}$, is regarded here as caused by the reaction $\alpha$-particles.

The ${ }^{7} \mathrm{Li}(\mathrm{d}, \mathrm{n} 2 \alpha)$ reaction rate $R_{\mathrm{m}}$ per an incident deuteron is calculated from the measured $\alpha$-particle yield $Y_{\alpha}$ as:

$$
R_{\mathrm{m}}=\frac{Y_{\alpha} / 2}{N_{\mathrm{d}} \times N_{\mathrm{Li}}} \times \frac{4 \pi}{\Delta \Omega},
$$

where $N_{\mathrm{d}}$ is the number of deuterons incident on a droplet, $N_{\mathrm{Li}}$ is the number of Li droplets in a run, and $\Delta \Omega$ is the solid angle of the detector. Although a pair of $\alpha$-particles is produced in every reaction, one of them is observed on the detector, while the other is stopped in the Li bulk. Therefore the number of the nuclear reaction is half the number of the detected $\alpha$-particles multiplied by $4 \pi / \Delta \Omega$.

The $R_{\mathrm{m}}$ is compared with the reaction rate $R_{\mathrm{c}}$ calculated under an assumption of continuous slowing down of the incident ions:

$$
R_{\mathrm{c}}=\int_{0}^{E_{\mathrm{in}}} n_{\mathrm{Li}} \frac{\sigma(E)}{(-\mathrm{d} E / \mathrm{d} x)} \mathrm{d} E,
$$

where $n_{\mathrm{Li}}$ is the Li density, $-\mathrm{d} E / \mathrm{d} x$ is the stopping power of the target, $E_{\mathrm{in}}$ is the incident energy of deuterons, and $\sigma(E)$ is the reaction cross section for solid $\mathrm{Li}[9]$ with $S=2100 \mathrm{keV} \cdot \mathrm{b}$ [10]:

$$
\sigma_{12}(E)=\frac{S_{12}}{E^{1 / 2}\left(E+E_{\mathrm{S}}\right)^{1 / 2}} \exp \left[-\pi\left(\frac{E_{\mathrm{G}}}{E+E_{\mathrm{S}}}\right)^{1 / 2}\right],
$$

The hypothetical reaction rate with a thermodynamic enhancement, $R_{\mathrm{e}}$, is expressed in the form of Arrhenius equation as follows:

$$
R_{\mathrm{e}}=R_{\mathrm{c}} \times \exp \left(-\frac{\Delta G}{k_{\mathrm{B}} T}\right)
$$

where $k_{\mathrm{B}}$ is the Boltzmann constant, $T$ is the temperature of the metallic $\mathrm{Li}$, and $\Delta G$ is the change in Gibbs free energy during transition from the "dilute solution of deuterium in the Li" to the intermediate complex [LiD] which is transformed adiabatically to the $\left[{ }^{8} \mathrm{Be}+\mathrm{n}\right]$ state through a transient ${ }^{9} \mathrm{Be}^{*}$ state. The exponential term in eq.(5) is the enhancement factor defined in Refs. 8 and 11. 


\subsection{Results and discussion}

${ }^{7} \mathrm{Li}(\mathrm{d}, \mathrm{n} 2 \alpha) \alpha$-particles having an energy of $5.7 \mathrm{MeV}$ after passing through the Ti filter foil would be detected in the range from 5 to $6 \mathrm{MeV}$. Here the assumed broadening of the spectral peak may possibly be due to inhomogeneous deposition of $\mathrm{Li}$ on the Ti foil and FWHM degradation of the SSD in the environment with an elevated temperature.

Two examples of the energy spectra recorded on the MCA are shown in Fig. 5. The spectra are dominated by ${ }^{241} \mathrm{Am} \alpha$-particles and electronic noise produced mainly in the ion source. We observe few counts, which could be electronic noise, in the energy range of interest (ROI) where the ${ }^{7} \mathrm{Li}(\mathrm{d}, \mathrm{n} 2 \alpha)$ reaction products would appear.

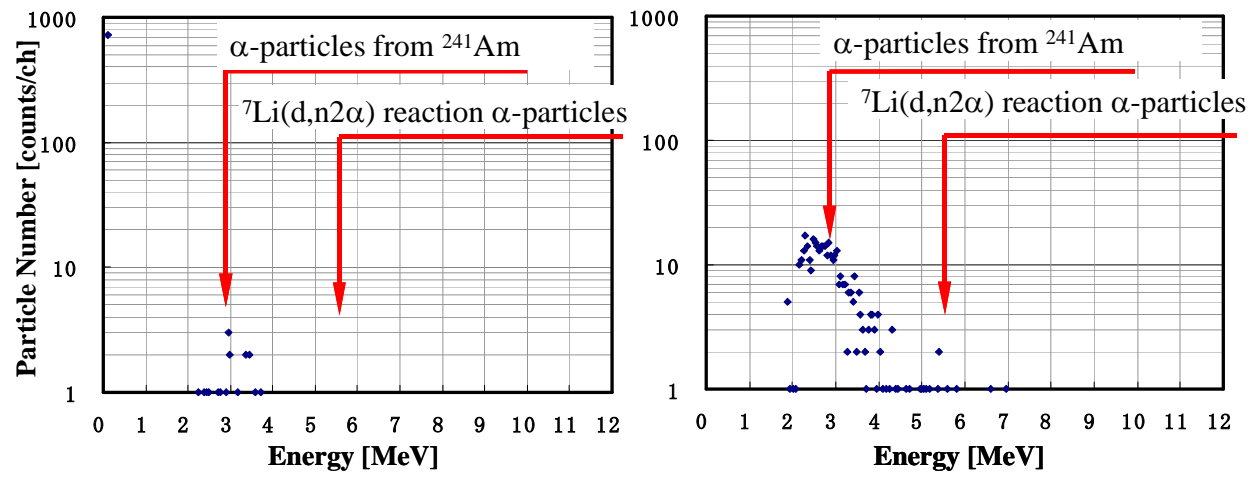

\begin{tabular}{|c|c|c|}
\hline $24.0 \mathrm{keV}-\mathrm{D}^{+}$ & incident particle & $10.0 \mathrm{keV}-\mathrm{D}^{+}$ \\
\hline $0.1 \mu \mathrm{A} / \mathrm{cm}^{2}$ & current density & $0.3 \mu \mathrm{A} / \mathrm{cm}^{2}$ \\
\hline $570 \mathrm{~K}$ & temperature of $\mathrm{Li}$ & $570 \mathrm{~K}$ \\
\hline $1.1 \mathrm{msr}$ & solid angle of SSBD & $1.1 \mathrm{msr}$ \\
\hline $10-\mu \mathrm{m} \mathrm{Ti}$ & filter & $10-\mu \mathrm{m} \mathrm{Ti}$ \\
\hline
\end{tabular}

Fig. 5. Two examples of $\alpha$ energy spectra.

Figure 6 shows energy dependence of the ${ }^{7} \operatorname{Li}(\mathrm{d}, \mathrm{n} 2 \alpha)$ reaction rate. Since there are few observed counts in the ROI, as seen in Fig. 5, it is rather difficult to distinguish the reaction $\alpha$-particles from the electronic noise. Assuming that only one count in the spectrum is ascribed to the ${ }^{7} \operatorname{Li}(\mathrm{d}, \mathrm{n} 2 \alpha)$ reaction product, the maximum value of $R_{\mathrm{m}}$ corresponding to the one count is expressed as the data point with a vertical line extending to null in Fig. 6. The data points are shown in the figure as an open circle ( $10 \mathrm{keV}, 570 \mathrm{~K})$, a closed circle (13 keV, $520 \mathrm{~K})$, an open triangle ( $20 \mathrm{keV}, 570 \mathrm{~K})$ and an open square $(24 \mathrm{keV}, 570 \mathrm{~K})$.

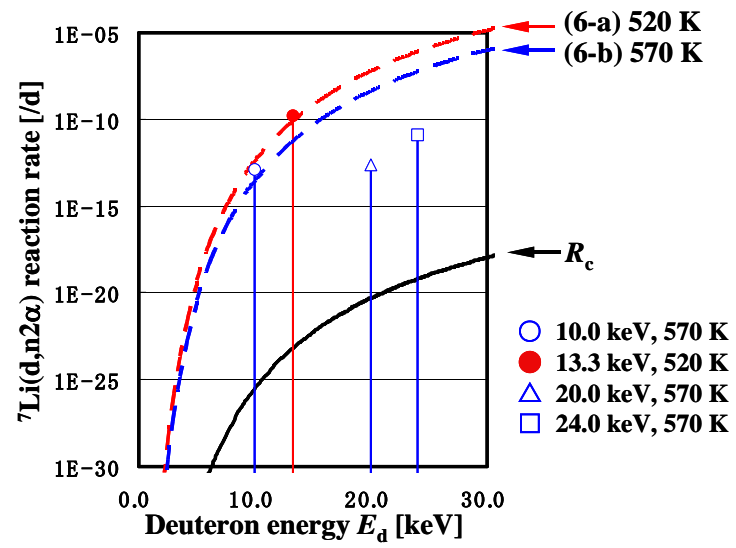

Fig. 6. Energy dependence of the ${ }^{7} \operatorname{Li}(\mathrm{d}, \mathrm{n} 2 \alpha)$ reaction rate. 
The solid curve shows the theoretical reaction rate $R_{\mathrm{c}}$. The broken curves (6-a) and (6-b) show the thermodynamically enhanced reaction rate $R_{\mathrm{e}}$ evaluated at the two different temperatures by using equation (5), where the value of the Gibbs energy change was assumed to be $-1.35 \mathrm{eV}$. [11]

Figure 7 shows the same data expressed as temperature dependence of the ${ }^{7} \operatorname{Li}(\mathrm{d}, \mathrm{n} 2 \alpha)$ reaction rate and the enhancement factor, $R_{\mathrm{m}} / R_{\mathrm{c}}$. The symbols are the same as those used in Fig. 6. In (7-b) the experimentally observed enhancement factor $R_{\mathrm{m}} / R_{\mathrm{c}}$ is compared with the enhancement factor, eq.(5).

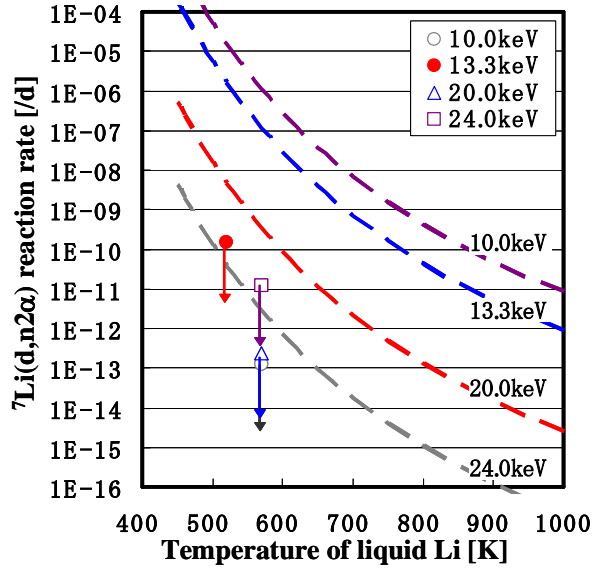

(7-a)

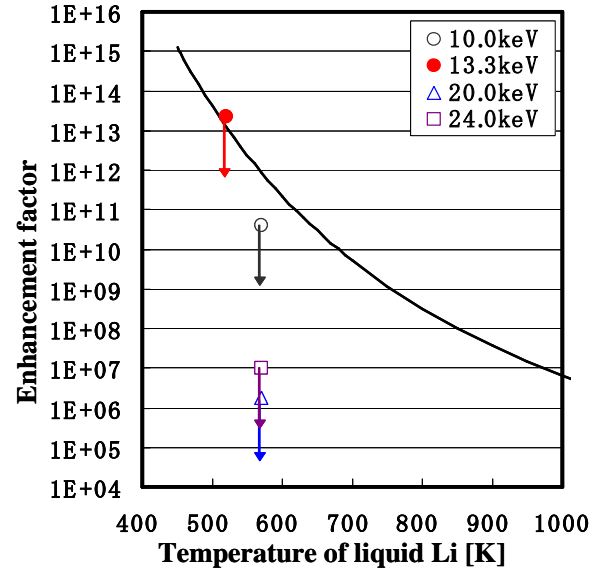

(7-b)

Fig. 7. Temperature dependence of the reaction rate (7-a) and the enhancement factor (7-b).

It is found that in all cases examined the enhancement factors are nearly equal to or much smaller than the thermodynamic enhancement factor. For example, the reaction rate $R_{\mathrm{m}}$ observed in the latest experiment for $20 \mathrm{keV}$ deuteron irradiation is less than $2.3 \times 10^{-13}$. On the other hand, the reaction rate $R_{\mathrm{c}}$ calculated with eq. (4) is $4.8 \times 10^{-21}$. The reaction rate enhancement $R_{\mathrm{m}} / R_{\mathrm{c}}$ is therefore $4.8 \times 10^{7}$ at the maximum, while according to eq. (5), the enhancement factor at a Li temperature of $570 \mathrm{~K}$ is expected to reach $8.6 \times 10^{11}$.

In summary the enhancement observed in the present work is much smaller than that claimed by $\mathrm{H}$. Ikegami et al. At least it could be said that the Ikegami enhancement, if any, is realized under rather specialized condition, and therefore difficult to reproduce.

\section{Summary}

We have described three kinds of experimental work at Kobe University: D(d,p)t reaction rate enhancement in deuterated $\mathrm{Au} / \mathrm{Pd}$ samples; PIXE analysis of Pd complex under $\mathrm{D}_{2}$ gas permeation; and ${ }^{7} \mathrm{Li}(\mathrm{d}, \mathrm{n} 2 \alpha)$ reaction rate enhancement in liquid lithium. The largest effort has been given to the last item, where we established the new experimental system capable of bombarding slag-free liquid Li droplets with mass-analyzed deuterium ion beams. Thus far, in all cases of irradiation at the temperature of $520-570 \mathrm{~K}$ with $10-24 \mathrm{keV}$ deuterons, we have not been able to reproduce the Ikegami enhancement for the ${ }^{7} \operatorname{Li}(\mathrm{d}, \mathrm{n} 2 \alpha)$ reaction. 


\section{References}

[1] A. Kitamura, Y. Awa, T. Minari, N. Kubota, A. Taniike, and Y. Furuyama; Proc. 10th Int. Conf. on Cold Fusion, Cambridge, MA: LENR-CANR.org.

[2] Y. Awa et al.; Proc. JCF5, December 15-16, 2003, Kobe University, Japan, pp.6-10.

[3] Y. Iwamura, M. Sakano and T. Itoh; Jpn. J. Appl. Phys. 41 (2002) 4642-4650.

[4] Evidence of Enhanced Nonthermal Nuclear Fusion; H. Ikegami, and R. Pettersson; Bulletin of Institute of Chemistry, BENF No.3 (Uppsala University, September 2002).

[5] J. Kasagi, H. Yuki, T. Baba, T. Noda, T. Ohtsuki and A. G. Lipson; J. Phys. Soc. Jpn. 71 (2002) 2881-2885.

[6] Effects of Electron Screening on Low-Energy Fusion Cross Sections; H.J. Assenbaum, K. Langanke and C. Rolfs; Z. Phys. A - Atomic Nuclei 327, 461-468(1987).

[7] J. Kasagi, H. Yuki, T. Itoh, N. Kasajima, T. Ohtsuki and A. G. Lipson; Proc. ICCF7(1998).

[8] Buffer Energy Nuclear Fusion; H. Ikegami; Bulletin of Institute of Chemistry, BENF No.1 (Uppsala University, September 2002): H. Ikegami, Jpn. J. Appl. Phys. 40(2001)6092.

[9] S. Ichimaru and H. Kitamura; Phys. Plasma 6(1999)2649, also refs. 15 and 16.

[10] Estimated from the data by C.H. Johnson and C.C. Trail; Phys. Rev. 133(1964)B1183.

[11] Enormous entropy enhancement revealed in linked nuclear and atomic $\mathrm{Li}+\mathrm{D}$ fusion in metallic $\mathrm{Li}$ liquid; $\mathrm{H}$. Ikegami et al.; Proc. FUSION03 Conference, Matsushima, November12-15, 2003. 